\title{
Germanica
}

\section{Heinrich Mann et Nietzsche ou la séduction de l'esthétisme}

Heinrich Manns Rezeption von Nietzsche: Reiz und Gefahr des Ästhetizismus

\section{Chantal Simonin}

\section{OpenEdition}

\section{Journals}

Édition électronique

URL : http://journals.openedition.org/germanica/2409

DOI : 10.4000/germanica.2409

ISSN : 2107-0784

\section{Éditeur}

Université de Lille

\section{Édition imprimée}

Date de publication : 30 juin 2000

Pagination : 57-68

ISBN : 9782913857032

ISSN : 0984-2632

\section{Référence électronique}

Chantal Simonin, « Heinrich Mann et Nietzsche ou la séduction de l'esthétisme », Germanica [En ligne], 26 | 2000, mis en ligne le 07 mars 2014, consulté le 06 octobre 2020. URL : http://

journals.openedition.org/germanica/2409; DOI : https://doi.org/10.4000/germanica.2409

Ce document a été généré automatiquement le 6 octobre 2020.

(ㄷ) Tous droits réservés 


\title{
Heinrich Mann et Nietzsche ou la séduction de l'esthétisme
}

Heinrich Manns Rezeption von Nietzsche: Reiz und Gefahr des Ästhetizismus

\author{
Chantal Simonin
}

1 Un lecteur non prévenu ne peut qu'être frappé à la fois par l'abondance et par le caractère hétéroclite de la production littéraire du «premier» Heinrich Mann, qui explique en partie le peu d'écho qu'elle ait reçu auprès du grand public jusqu'à l'édition massive, en 1918, de Der Untertan qui valut à son auteur son premier - et dernier immense succès de librairie. Il est étonnant en particulier de voir comment, dans les œuvres qui s'échelonnent de 1895 à 1905, les styles se suivent et ne se ressemblent pas, comment l'auteur adopte telle manière pour s'en détacher, un an après, et en adopter une autre, radicalement différente. En décembre 1901, à l'époque où il écrit Die Göttinnen, H. Mann écrit à son ami lübeckois Ludwig Ewers qu'il vient d'être contacté par un journal d'Innsbruck, qui lui demande des contributions, avec l'idée sans doute, "ce qui est une erreur", qu'il continue d'écrire dans le style de Im Schlaraffenland, terminé en mars 1900. En juillet 1905, il écrit à son amie Agnès Schmied, qui vient de terminer la lecture de Professor Unrat rédigé pendant l'année 1904, qu'il se sent dans une certaine mesure proche de Unrat, alors qu'il n'a pour ainsi dire plus aucune affinité avec tout ce qui se trouve dans Die Göttinnen (WL, 107).

On a donc affaire ici à un artiste qui cultive l'éclectisme, à un auteur caméléon qui revêt successivement des identités d'emprunt avec lesquelles il se plaît à jouer, non pas tant pour masquer son être véritable que pour donner de la consistance à une vie qui, fondamentalement, en est dénuée: attitude frivole propre au dilettante avide de collectionner les «sensations » qui lui permettront de vivre plus intensément, et de pimenter une existence avant tout perçue comme radicalement solitaire. Cette idée que l'artiste est voué à une solitude aristocratique, très répandue dans les milieux littéraires à la fin du xix siècle chez les dandys entichés de snobisme, et dans laquelle on reconnaît sans peine l'influence de la définition aristocratique de la culture énoncée par Nietzsche, va prendre chez H. Mann une signification personnelle de plus en plus douloureuse. Ce qui relevait d'abord de la pose et d'une certaine convention littéraire 
devient, au fil des années, conscience d'un manque. Une notice autobiographique de 1904 expose avec une certaine lassitude comment un créateur privé d'un public " doté des mêmes instincts » que lui, vivant dans une solitude que vient renforcer dans son cas l'absence de véritable patrie puisqu'il est d'ascendance germanique par son père et latine par sa mère, est contraint de se construire un monde à soi, de se replier sur sa propre solitude et de la savourer en l'agrémentant d'ingrédients plus ou moins corsés, tout en cultivant un individualisme qui semble bien être perçu comme une impasse: «Comme il n'existe nulle part de public doté d'instincts exactement semblables, on en vient à réduire son cercle d'influence, et à ne plus tenir compte que de soi seul... On se procure à soi seul des sensations. On cherche à vivre plus intensément sa propre vie, à pimenter sa propre existence » (Diana, 346).

3 Six ans plus tard, le ton a changé : une nouvelle notice autobiographique de I9I0 propose un bilan établi par un auteur ayant atteint le seuil de l'âge mûr et qui mesure avec assurance le chemin parcouru entre "l'affirmation de l'individualisme » tel qu'il s'est encore exprimé dans Die Göttinnen, et l'hommage rendu dans Die kleine Stadt (1907) à la démocratie, au « peuple ", à l'« humanité » (WL 122). Entre temps, Heinrich Mann a surmonté une crise au terme de laquelle il rejette avec véhémence une conception élitiste et individualiste de l'art et de la culture ainsi que ceux qui furent jusqu'alors les grands modèles, Flaubert et Nietzsche.

Incontestablement, H. Mann a lu Nietzsche avec passion. Une notice de mai 1892 nous apprend que la lecture de Nietzsche constituait alors sa «lecture principale » (WL, 70). Et dans ses mémoires, intitulées Ein Zeitalter wird besichtigt, H. Mann rend hommage, avec circonspection, à Nietzsche en qui il salue successivement «le bon Européen ", "l'adversaire farouche du "Reich» (qu'il (Nietzsche) n'évoque qu'avec des guillemets)» et «le disciple des Moralistes français». La discrétion qu'il observe par rapport à l'emprise temporaire et à l'influence durable qu'a exercé sur lui le grand philosophe s'explique par des raisons très personnelles: l'évolution de l'histoire allemande ne pouvant que le rendre sceptique quant à la réussite de son combat démocratique, H. Mann se montre au fil des ans de plus en plus soucieux, sans doute pour opposer à la réalité ses " convictions ", de laisser une image de lui qui coïncide au plus près avec l'image idéale de l'écrivain qu'il portait en lui-même, celle du "grand homme démocratique " cher à son cœur, qu'il avait longuement élaborée, non sans retours difficiles sur lui-même, dans les essais qu'il consacre à la littérature française entre 1905 et 1910. Ses mémoires publiées en 1945, ainsi que la correspondance avec Karl Lemke qui projetait en 1946 d'écrire une biographie d'H. Mann et lui demande alors de lui fournir quelques indications susceptibles de l'aider, nous livrent de lui un portrait quelque peu retouché : aucune allusion à la revue conservatrice Das zwangzigste Jahrhundert que H. Mann édite d'avril 1895 à mars 1896, depuis l'Italie où il séjourne alors, ni au tout premier roman intitulé In einer Familie, rédigé entre août 1892 et octobre 1893 et s'inspirant de la pensée conservatrice de Paul Bourget auquel il était dédié, de même peu ou pas d'allusions à Nietzsche, dont la pensée a connu dans l'idéologie nazie des prolongements fâcheux.

5 C'est dans la trilogie Die Göttinnen que s'affirme avec le plus d'éclat "l'individualisme » que H. Mann estimait, en 1910, avoir dépassé et que Nietzsche, en 1890, incarnait à ses yeux de manière exemplaire. "Nous avions une foi joyeuse en celui qui représentait l'extrême pointe de l'individualisme et qui aurait été anarchiste plutôt que sujet soumis du Reich» (Nietzsche, 8). La trilogie, rédigée du début de l'année 1900 au mois de 
septembre 1902, met en scène une "personnalité belle, libre, avide de jouissances " (WL, 122) et raconte les aventures extravagantes, tumultueuses d'une " grande dame de Dalmatie ", qui pratique, elle aussi, le dilettantisme, puisque le récit la mène successivement à Rome où la duchesse Violante von Assy fomente par désœuvrement une conspiration qui va échouer, - c'est le roman intitulé Diana -, puis à Venise où elle s'adonne à toutes les jouissances artistiques - Minerva -, à Naples enfin où, devenue une femme vieillissante, elle savoure sans retenue toute la palette des plaisirs charnels et sensuels: il s'agit là du troisième tome intitulé Venus. Capitale pour la compréhension du personnage est la notion d'intensité (Lebensgefühl) associée à celle de "Renaissance »: bien que l'histoire se déroule dans l'Italie du XIX ${ }^{e}$ siècle, H. Mann a qualifié son roman de « roman de la nouvelle Renaissance ». L'héroïne, au narcissisme exalté, est une incarnation de l'«Esprit», par quoi il faut entendre une forme d'intelligence prête à toute les audaces, à toutes les jouissances, à tous les " débordements " (" Ausschweifungen »), terme qui revient constamment dans l'œuvre de H. Mann. Figure féminine audacieuse, elle annonce Rosa Fröhlich qui subjugue Unrat par "la puissance de sa volonté", ainsi que l'héroïne du tout dernier roman de Heinrich Mann, publié en 1949 et totalement abscons : la baronne Kovalsky, comtesse de Traun, «figure internationale » que ses activités de résistance pendant la seconde guerre mondiale ont rapprochée d'un ouvrier communiste français répondant au nom de Vertugas avec lequel, » «si elle avait vécu, elle aurait pris l'avion pour Moscou en $1939 »(\mathrm{WL}, 338)$.

6 Le contenu du roman est délibérément rutilant : dans une lettre datée du 2 décembre 1900, Heinrich Mann annonce à son éditeur Albert Langen que, si tout se passe bien, la première partie de sa trilogie sera "parée de couleurs exotiques", la seconde «lourde de toutes les ivresses de l'art ", la troisième "obscène et amère ». Le style privilégie l'outrance, le maniérisme, et le lecteur d'aujourd'hui ne peut s'empêcher d'éprouver un certain malaise face à cet esthétisme de pacotille : derrière cette avalanche de décors somptueux, ce personnage féminin aux toilettes richement ornementées, et dont la sensualité exigeante s'entoure de jeunes éphèbes complaisants, on sent trop un auteur préoccupé de fabriquer du beau et de «faire riche» au détriment de la substance du livre. Les sources littéraires dont H. Mann s'est inspiré pour tracer l'image qu'il entendait donner de l'Italie sont d'ailleurs révélatrices : Burckardt, Théophile Gautier, Stendhal, Taine, Henri de Régnier. Et pour écrire ce qui devait être sa "Salambo " (Banuls), Heinrich Mann avait puisé sans vergogne dans des chroniques journalistiques de l'époque relatant la vie plus ou moins scandaleuse de personnages en vue, aristocrates sur le déclin, qui l'approvisionnaient en potins mondains : la «Chronique scandaleuse de Rome » et le « Wiener Hofklatsch» (Diana, 317).

7 Le livre est donc un peu un fourre-tout, il a du reste agacé une certaine partie de la critique, et en tout premier chef Thomas Mann qui, dans ses Betrachtungen, s'en prend violemment à la mode de la Renaissance et à ce «nietzschéanisme » de mauvais aloi, issu d'une lecture superficielle et naïve de ce philosophe dont nombre d'écrivains, avides de se procurer des sensations à vil prix, se sont entichés au tournant du siècle en produisant « des romans chargés de festivités où l'on célébrait la beauté, des romans à l'inspiration pubertaire et aphrodisiaque, des catalogues de vices répertoriés au grand complet » (Diana, 351). Incontestablement, Heinrich Mann a commis, avec ses Göttinnen, un ouvrage de cette veine : le troisième volume en particulier, qualifié par H. Mann de «roman du climactérium» (mot savant pour désigner ce qu'il est convenu d'appeler l'âge critique de la femme) abonde en évocations érotiques un tantinet scabreuses, mais 
il serait injuste et faux de ne retenir que ce côté outrancier, sans doute le plus directement accessible de l'œuvre. Il importe au contraire d'en saisir l'intention première dont elle procède, ce qui, derrière cet esthétisme aujourd'hui démodé, lui confère un caractère authentique: en voulant donner forme à une réalité plus « intense » que celle de l'expérience quotidienne et banale, le romancier s'oppose de manière véhémente à la médiocrité et à la platitude bourgeoise qui l'entourent. "Accablé par les bourgeois qui pullulent dans mon pays, je me transporte en Dalmatie »: c'est ainsi que H. Mann présente à son éditeur Albert Langen, dans une lettre du 2 décembre 1900, le nouveau roman dont il est en train de tracer les grandes lignes. (Diana, 299). Cette charge contre le bourgeois philistin, dans laquelle on reconnaît sans peine l'influence de Flaubert et de Nietzsche, n'est pas nouvelle : en 1889 déjà, H. Mann, alors âgé de dix-neuf ans, se moquait dans une petite nouvelle au contenu iconoclaste, intitulée Fantaisie à propos de ma ville natale L. des «odeurs mercantiles » qui infestaient Lübeck, sa ville natale. (WL, 12). Quinze ans plus tard, on retrouve dans Professor Unrat, par delà ce vaste détour qu'ont constitué Die Göttinnen, la même aversion tenace pour la petite ville hanséatique où il a grandi, cette espèce de "nid de bourgeois", dans lequel des marchands stupides, uniquement soucieux de s'enrichir, tiennent le haut du pavé.

8 Professor Unrat, publié en 1905, donc deux ans à peine après Die Göttinnen, change radicalement de registre en sorte que son auteur, on l'a vu, avait le sentiment de n'avoir plus grand-chose en commun avec l'héroïne de sa trilogie. Le livre eut peu de succès, et c'est le film de Sternberg qui l'a fait connaître au grand public. Depuis, la critique littéraire s'en est emparée en insistant beaucoup sur la satire de la société wilhelminienne qui transparait derrière l'intrigue. Ceci pourtant ne rend compte que d'un aspect du roman. Une lecture plus approfondie permet de mesurer à quel point H. Mann hésite ici entre plusieurs conceptions de la littérature, aussi bien sur le plan stylistique que dans l'approche du sujet qu'il a décidé de traiter, et combien ce roman, écrit en quelques semaines seulement, marque une transition entre l'esthétisme qu'il a défendu jusqu'alors et qui continue de le séduire et un engagement démocratique qui ne sera explicitement revendiqué qu'en 1910, au moment des déclarations fracassantes de l'essai intitulé Geist und Tat.

Peu d'études se sont penchées sur le personnage du roman qui constitue l'adversaire sur lequel Unrat s'acharne de manière quasi hystérique. Dandy intelligent, nouvelle figure de dilettante, l'élève Lohmann apparait bien comme un frère cadet de H. Mann qui adopte envers lui une attitude où l'identification se mêle à l'auto-dérision. Avec sa chevelure sombre, la mèche "mélancolique " qui lui barre le front, sa "pâleur de Lucifer ", ses ongles soignés et ses talents de mime, ce jeune aristocrate, un peu plus âgé que ses condisciples, forme un contraste parfait avec son ami von Erztum, jeune junker massif et bête, homme de la glèbe, puissamment sensuel et fleur bleue. Sa supériorité intellectuelle et sociale en fait le contraire du type du parvenu - tel que l'incarne Unrat lui-même, avec son chapeau gras et les pellicules qui recouvrent ses épaules, ou l'écolier Felix dans la nouvelle Abdankung, Diedrich Hessling dans Der Untertan et, après lui encore, les "ratés » de tout acabit qui cautionnent la dictature hitlérienne. Contraints d'assister au «triomphe de Unrat» émergeant, l'air complètement rajeuni, dans une tenue débraillée, de la loge de Fröhlich, les trois élèves présents, en proie aux affres de la puberté et tous d'une manière ou d'une autre intéressés par l'« actrice ", réagissent différemment. Dilettante nonchalant, Lohmann refuse de porter un jugement moral sur la situation et c'est à lui que revient l'honneur 
de formuler la thèse à la fois subtile, pompeuse et désuète qui sous-tend le roman, celle qui cherche à cerner le " cas Unrat » selon laquelle le «tyran » est un anarchiste en puissance.

H. Mann évoque avec une tendresse amusée ce jeune double de lui-même. Derrière la carapace d'ironie et l'attitude aristocratique du jeune dandy se cache un amoureux transi. Lohmann aime en secret une femme plus âgée que lui, l'épouse du consul Breetpoot, au nom bien ancré dans l'épaisse réalité bourgeoise, qui lui inspire des tourments évoqués par l'auteur avec un luxe de raffinements, tout comme cette « envie sauvage » que Lohmann éprouve pour Rosa Fröhlich, « cette envie qui le poursuit, cette envie d'humilier son amour cruel par les sombres caresses du vice» (109). La fin du roman complète ce portrait gentiment ironique. L'on y voit réapparaître Lohmann mûri, désillusionné. Confortablement installé dans une pâtisserie de la ville, aux côtés de Rosa Fröhlich rencontrée par hasard, l'esthète et " analyste » qu'il est resté expose avec un plaisir évident devant la jeune femme devenue entre temps une "demimondaine de grand style " l'idée qu'il se fait de Unrat, ce tyran qui, pour peu que les circonstances lui résistent, " convoque la plèbe dans le palais, l'incite à tout mettre à feu et à sang et proclame l'anarchie » (176). Mais lorsque, quelques heures plus tard, Unrat les surprend ensemble et veut égorger sa femme, Lohmann, confronté à une réalité qui n'a plus rien à voir avec l'hypothèse abstraite qui le séduisait, a le réflexe tout bête et «bourgeois » d'appeler la police (184). Réaction de bon sens qui jette une lumière légèrement cruelle et ironique sur les échafaudages intellectuels du jeune dandy. Il reste que H. Mann adhère lui-même à la thèse développée par Lohmann, même si elle est présentée, dans les toutes dernières pages du roman, comme un jeu intellectuel frivole et parfaitement abstrait. Et c'est avec lyrisme qu'il évoque, dans une page spectaculaire qui multiplie les effets rhétoriques et les images précieuses, le moment où Unrat se délecte d'avoir réussi à faire sombrer dans le vice la ville tout entière : «Et cette déchéance morale de toute une ville, à laquelle personne ne pouvait mettre un terme parce que trop de monde y était impliqué : c'était le fait de Unrat et de son triomphe. C'est une ville entière qui payait un tribut et qui succombait à sa passion - à cette passion qui l'habitait et dont son corps desséché ne laissait rien paraître, à part de temps en temps un éclair qui brillait dans son regard vert et venimeux, ou encore un ricanement blême. » (163-164)

11 La préciosité, envahissante ici, n'exclut pas le recours au grotesque, on peut même dire qu'elle l'appelle et tout permet au contraire d'affirmer que le recours à ce registre, si fréquent chez H. Mann, constitue pour lui un détour paradoxal de l'esthétisme, une autre manière de fuir et de transcender le réel. Dans la lettre, déjà citée, du 2 décembre 1900 à son éditeur Albert Langen, H. Mann évoque les personnages qui entourent la duchesse d'Assy et qui sont, dit-il, des animaux réjouissants comme ceux que j'ai dépeints dans Im Schlaraffenland et il ajoute: "il se passe en Dalmatie, à la cour de Nikolaus von Koburg, "des choses grotesques qui m'élèvent au-dessus du quotidien" !» (Diana 300). Les effets grotesques auxquels il recourt si volontiers et dans lesquels s'exprime un goût de l'audace en partie imputable selon lui à ses origines semiexotiques (WL, 494), vont de pair chez lui avec une tendance évidente à l'abstraction. C'est vrai pour Unrat, c'est vrai aussi pour Der Untertan où l'on relève, après les trois premiers chapitres qui livrent un portrait psychologique magistral de Diederich Hessling, nombre d'invraisemblances qui rendent ingrate et malaisée la lecture de l'ouvrage. Les romans de la vieillesse enfin, dans lesquels Thomas Mann a salué, non sans quelque embarras, "l'avant-gardisme d'un vieillard», (à propos de Der Atem) 
présentent des personnages évoluant dans une réalité aux contours très schématiques. Conçu comme une fin en soi plutôt que comme un moyen d'appréhender une réalité politique et sociale, le recours au grotesque, tel qu'il se manifeste de façon quasi constante chez H. Mann, semble bien être la trace, une trace qui s'amenuise au fil des années, d'une conception de la littérature qui voit dans l'art un narcotique, un moyen de parvenir à l'« ivresse ».

12 À mi-chemin entre le roman et la fable, Professor Unrat ne débouche pas sur un constat politique et social précis. Mais le reproche d'abstraction ne vaut que pour la thèse qui sous-tend le roman. Concrètement, le roman propose un portrait, celui d'un type psychologique précis que $\mathrm{H}$. Mann s'applique à disséquer dans ses composantes les plus intimes, en jetant sur son personnage, tout comme l'élève Lohmann qui, en raison du "penchant qu'il éprouve à observer les raretés humaines", se sent "vivement concerné » par le " cas Unrat ", un regard qui est à la fois celui d'un moraliste et d'un entomologiste. Ce faisant, il s'inspire d'un aspect plutôt méconnu de l'œuvre de Nietzsche : l'entreprise de démystification à laquelle s'est livré le philosophe. Formé, tout comme H. Mann, à l'école de l'analyste Paul Bourget, rompu aux subtilités de l'analyse psychologique à travers la lecture de La Rochefoucauld et de Stendhal, Nietzsche s'est appliqué a disséquer les comportements humains. Soucieux de faire émerger "la partie honteuse $»^{1}$ de notre monde intérieur, il aborde la morale traditionnelle avec les outils du " psychologue » ou même du " physiologue ", affirmant la nécessité d'utiliser des termes "zoologiques" pour exprimer au plus juste des réalités morales qui rendent compte des comportements de l'« espèce humaine » (II, 979). H. Mann adopte la même attitude clinique et se livre, en faisant le portrait de son vieux professeur à une véritable vivisection, celle d'un "type " d'individu. Ce type, d'abord psychologique, devient, au fur et à mesure que H. Mann s'affirme comme auteur politique, le représentant d'une catégorie sociale, celle du petit-bourgeois imbécile, pusillanime et violemment conservateur. Tout se passe comme si, à partir de 1904, Heinrich Mann cherchait méthodiquement, à travers des approches successives, à cerner de l'intérieur, avec le maximum de proximité possible, au point d'épouver cette proximité comme une complicité quelque peu répugnante, une réalité psychologique, sociale et politique qu'il abhorre pour mieux mettre sur pied, en le définissant a contrario, un idéal qualifié de «bouleversant » et qui sera un idéal démocratique. Il est facile de constater avec quelle continuité se succèdent dans son œuvre Unrat, l'écolier Felix, figure de " tyran » lui aussi dans la nouvelle Abdication, le sujet Diederich Hessling ressenti comme " répugnant et intéressant ", Allemand "moderne " par excellence, et préfiguration de l'Allemand nazi, dont $\mathrm{H}$. Mann fait le portrait dans son premier recueil d'essais antinazis, intitulé La haine, qui paraît en 1933, en français d'abord chez Gallimard, avant d'être traduit en allemand par H. Mann lui-même. Le dilettantisme et la littérature d'analyse dont H. Mann prend congé dans Professor Unrat, non sans difficulté comme le montrent les sentiments contradictoires que lui inspire le jeune Lohmann, a développé en lui une acuité psychologique qui, au lieu de s'exercer de manière gratuite et frivole, se met peu à peu au service d'une réflexion politique et morale.

13 Cette perspective n'est pas encore explicite dans Professor Unrat, elle existe seulement en filigrane. Le roman, sans présenter encore de personnage positif comme ce sera le cas dans Der Untertan peut se lire comme la condamnation, à travers la ruine du vieux tyran, d'une certaine forme de nihilisme quasi-suicidaire. La perversité du tyran est l'envers de la bonté et de l'humanité de l'individu normalement constitué. D'ailleurs, si 
l'on y regarde de près, Unrat n'est pas vraiment antipathique : en dépit de ses accès de fanatisme, on a du mal à voir en lui un monstre. C'est aussi un vieil homme solitaire ayant lui-même confusément conscience d'être un hurluberlu suspect, vivant en marge de la société. Coupé de toute vie sociale, usé par le combat qu'il mène contre le monde entier, il trouve dans le cabaret de l'Ange Bleu un monde après tout chaleureux où, pour la première fois, il a le sentiment d'être un être humain comme les autres et où il peut, en faisant abstraction du «monde récalcitrant » qui l'entoure, enfin s'abandonner ou encore « abdiquer ». L'actrice Rosa Fröhlich, au visage barbouillé de couleurs hurlantes, provoque chez lui un accès de gentillesse tout à fait nouveau. Lorsque, constatant qu'il " n'a pas d'idées derrière la tête ", elle éclate en sanglots pour brusquer les choses : « Je vais essayer de vous faire passer ", lui répond Unrat avec émotion. Ces mots, commente le narrateur, «qu'un professeur aurait pu dire à un élève qu'il aimait bien menacé de redoublement, Unrat ne les avait encore jamais dits à personne » (82). Et lorsque Unrat apprend, lors du procès où sont jugés les trois élèves qui ont profané une tombe qui remonte à l'époque des Huns, que l'élève Kieselack a eu des relations intimes avec l'actrice, il n'est plus alors qu'un vieil homme pathétique et sanglotant pour lequel le lecteur éprouve, non plus la sympathie qu'inspire à Lohmann l'«anarchiste intéressant ", mais une véritable compassion.

14 Le personnage de Unrat, que le roman s'applique à présenter sous divers éclairages, accuse du reste quelque ressemblance paradoxale avec son auteur : la solitude du tyran barricadé dans ses pulsions de ressentiment est, toutes proportions gardées, comparable à la solitude matérielle et affective dans laquelle H. Mann s'est enfermé en cultivant la pose du dandy aristocratique. Le jeune artiste dilettante qui rêvait en 1893 de pouvoir s'offrir un mois d'une «existence supérieure " qui se déroulerait à Paris (WL, 56) et qui, fuyant l'Allemagne wilhelminienne, s'était précipité en Italie, croyant ainsi retourner dans ce qu'il pensait être son " pays natal ", se trouve, au fil des ans, en proie à un sentiment croissant d'isolement. En 1904 déjà, il confesse à son éditeur Albert Langen que, lassé de l'existence instable qu'il mène depuis dix ans, il aimerait bien s'acheter "une petite maison quelque part», tandis qu'en 1905, il reconnaît explicitement que les positions esthétiques affichées dans Die Göttinnen, en particulier « la grande sensualité païenne » qu'il y célébrait n'était jamais qu'un substitut, auquel il se raccrochait d'autant plus bruyamment qu'«il en était moins convaincu intérieurement » (WL, 106). Manifestement, H. Mann étouffe dans le monde personnel qu'il s'est construit et où il espérait se sentir à l'aise.

Contrairement aux attentes qu'il avait suscitées, l'esthétisme est, paradoxalement, une impasse. Alors que la conception de l'art à laquelle H. Mann s'était rallié jusqu'alors impliquait une certaine part de frivolité, c'est au nom même de cette frivolité qu'il commence, à partir de 1907 environ, à rejeter l'esthétisme synonyme selon lui d'indifférence politique. C'est la première fois en tous cas que l'on trouve sous sa plume, au moment où il commence à rédiger Die kleine Stadt, l'amorce d'un engagement démocratique qualifié de "radicalisme de l'Esprit». Rompant définitivement avec les positions nihilistes de Nietzsche, Heinrich Mann a voulu, en écrivant Die kleine Stadt, dont la rédaction s'échelonne de l'automne 1907 au 31 mars 1909, construire un monument en l'honneur du "peuple» et de l'«humanité ». De nouveau, l'action se passe en Italie, qui n'est plus une Italie mythique, vue à travers le prisme d'une série d'influences littéraires, mais bien l'Italie où l'auteur séjourne alors, à un moment où, cessant de jeter sur sa patrie d'élection le regard d'un esthète avant tout séduit par "des couleurs et par des formes ", il s'intéresse de plus en plus à ce qui l'entoure et 
regarde vivre le petit peuple italien à partir duquel il conçoit les personnages de son nouveau roman. Une notice de 1907 nous éclaire sur les raisons de cet intérêt croissant et propose de ce même peuple une définition un peu courte, visiblement formulée par un intellectuel en mal de simplicité et décidé à observer ce qui l'entoure avec indulgence : «La musique italienne, de même que le peuple italien, m'apaisent. Cette animalité naïve est pour moi un contrepoids à mon intellectualité. Car j'ai sacrifié à mon intellectualité et je n'ai utilisé ma sensualité que pour nourrir en moi la vie de l'Esprit. Ici, je trouve des êtres qui me sont apparentés, et qui sont pour moi comme de jeunes frères, encore innocents » (WL, 115). En se tournant ainsi vers une réalité qu'il ignorait jusqu'alors, Heinrich Mann « abdique » à sa façon. Cette image sommaire mais nouvelle du peuple italien constitue une transition entre l'image de la «ménagerie ", c'est-à-dire du public imbécile, imbibé d'alcool, abruti par les accords assourdissants du piano, qui fréquente l'Ange Bleu et l'image sublimée du peuple français que H. Mann trace dans ses essais de 1910 où il s'enflamme pour le peuple français qui fut capable de remporter la victoire à Valmy, une victoire qui est celle de l'« Esprit » qui triomphe de la nature et de la tradition, une victoire que Voltaire aurait remportée contre Goethe (Voltaire - Goethe, 17).

16 À première vue, cet engouement pour la France et pour la démocratie a de quoi surprendre. Nul doute que c'est le moyen pour H. Mann de mettre fin à cette interrogation narcissique et douloureuse sur l'identité de l'artiste qui jalonne jusque-là sa production littéraire : l'art n'est plus une activité ludique, mais une «mission ». On est bien sûr tenté de voir dans cette conscience politique nouvelle une autre facette du dilettantisme professé jusqu'alors, largement tributaire, par le caractère outrancier du langage dans lequel elle s'exprime, d'un esthétisme dont H. Mann n'arrive pas à se défaire. C'est dans des affirmations grandiloquentes qu'il conclut son essai VoltaireGoethe: "La liberté est l'amour de la vie, qui inclut la mort. La liberté est la danse de ménades de la raison. La liberté est l'homme absolu » Et Thomas Mann parle dans ses Betrachtungen de l'«engagement démocratique infâme et hystérique " qui aurait succédé chez son frère à la célébration hystérique de la Renaissance. Tout ceci, heureusement, va se décanter. La véhémence des années 1910 - 1914, à travers laquelle $\mathrm{H}$. Mann accède douloureusement à une nouvelle conscience de soi, fait place à un ton plus modéré. Aux quelque quinze " années de formation », au cours desquelles H. Mann se nourrit d'influences littéraires diverses, sorte de terreau qui va féconder l'œuvre de la maturité, succèdent les années consacrées à ce qu'il appelle "le travail ». On aurait tort toutefois d'imaginer qu'il existe une rupture entre son esthétisme et son engagement démocratique. Bien sûr, il prend le contrepied des thèses nihilistes de Nietzsche puisque, s'il s'en prend aux différents régimes qui se succèdent en Allemagne dans la première moitié du vingtième siècle c'est pour leur opposer vigoureusement l'image d'une nation allemande idéale qu'il cherche à construire. Mais en opposant ainsi ce qui est à ce qui doit être, il adopte une démarche qui, dans son esprit, reste fidèle à Nietzsche puisqu'il refuse de tomber dans ce travers typiquement allemand selon Nietzsche qui consiste à se laisser obnubiler par les faits établis et qu'il fait du même coup preuve de cette indépendance d'esprit prônée par le philosophe. Sur le fond aussi, les analyses politiques de H. Mann font apparaitre des catégories vitalistes, irrationnelles, donnant lieu à des aperçus déroutants qui marquent une influence durable, définitive même de l'esthétisme de Nietzsche et de sa théorie de la décadence : l'idée par exemple que tout, dans le Reich allemand, est, en dépit du dynamisme officiellement affiché, synonyme de stérilité, affaire d'«imitation» et de 
« cabotinage », de théâtralité, qu'il s'agisse du sujet ou de l'Empereur Guillaume II, figure de "parvenu» auquel d'autres succéderont dans l'histoire allemande du vingtième siècle. Ces catégories concernent aussi bien les individus que les peuples ou les grandes phases de l'histoire. À l'opposé, il existe des périodes « inspirées », qui se caractérisent par l'intensité de leur vécu («Lebensgefühl») : la France d'Henri Quatre, la Révolution française, la Troisième République... Ces vues personnelles, parfois choquantes si elles sont évoquées de manière trop allusive, à travers une image dans laquelle se condense toute une réflexion qui échappe au lecteur naïf, viennent rehausser, pimenter en quelque sorte une pensée qui est avant tout une somme de " convictions" au nom desquelles Heinrich Mann a choisi de se battre. Ce n'est sans doute pas un hasard si c'est précisément à cette notion d'intensité que Klaus Mann a recours pour caractériser, dans son livre de mémoires, la pensée politique de son oncle, dont il partageait, dans ses années d'exil, la sensibilité politique : « La pensée politique de H. Mann a l'intensité, la véhémence authentique qui vient des tripes, qui vient du cœur ». Ici, la vie et la pensée ne font qu'un. La démocratie est aussi belle que juste. En se battant pour elle, $\mathrm{H}$. Mann mène un combat « inspiré », dans lequel il a le sentiment de s'accomplir. Ayant su conjurer ces dangers inhérents à l'esthétisme que sont l'isolement et la frivolité, sans pour autant renoncer aux émotions qu'il procure, il éprouve enfin l'intensité d'un vécu qui, parce qu'il fait appel à l'affectivité et à la raison, répond à ses aspirations profondes et généreuses que la pose du dandy avait longtemps refoulées, celles d'œuvrer, à l'instar des écrivains français qu'il admirait, comme un " grand homme démocratique ».

\section{BIBLIOGRAPHIE}

Heinrich Mann : 1871-1950. Werk und Leben in Dokumenten u. Bildern (Ausstellungskatalog); hrsg. von d. Dt. Akad. d. Künste zu Berlin, Weimar : Aufbau Verl., 1971. (WL dans l'article)

Mann, H. : Diana, Frankfurt/Main : Fischer, 1987. (Fischer TB ; 5925)

Mann, H. : Professor Unrat, Reinbek bei Hamburg : Rowohlt, 1985. (rm ; 35)

Nietzsche F. : Les Pages immortelles de Nietzsche, choisies et expliquées par H. Mann, Paris : Éditions Correa, 1985.

\section{NOTES}

1. En français dans le texte de Nietzsche. 


\section{RÉSUMÉS}

Publié en 1905, Professor Unrat, le texte de H. Mann le plus fameux avec Der Untertan, a été généralement compris comme une satire de la société wilhelminienne, proche dans ses intentions du mouvement expressionniste qui voyait alors le jour en Allemagne. Une lecture attentive du texte fait apparaitre une large composante autobiographique: l'auteur traverse une crise douloureuse, au terme de laquelle il se détache d'un esthétisme nourri par une lecture intensive de Nietzsche, depuis quelques années très à la mode en Allemagne, pour définir des positions personnelles, une nouvelle conception de l'art étroitement liée à un engagement politique démocratique qui détermine toute sa production ultérieure. La manière ambiguë dont l'auteur se situe par rapport à son personnage de «tyran » et à la fable qu'il développe montrent que Professor Unrat se situe exactement à mi-chemin entre les deux optiques, dont la seconde va prévaloir, sans que Nietzsche, le grand modèle du «premier» H. Mann, soit pour autant définitivement renié.

Professor Unrat, 1905 veröffentlicht und heute noch neben Der Untertan das berühmteste Werk von H. Mann, ist oft als eine Satire des deutschen Wilhelminismus verstanden worden und in der Anlage mit dem Expressionismus verwandt, der zur gleichen Zeit in Deutschland entstand. Eine genaue Untersuchung des Romans zeigt, dass er stark autobiographisch geprägt ist. Der Autor machte damals eine schwere Krise durch, in der er sich von dem Ästhetizismus distanzierte, den er bislang hemmungslos praktiziert hatte und der durch eine intensive Lektüre der Werke Nietzsches genährt worden war, um persönliche Positionen allmählich zu definieren, eine neue Auffassung der Kunst, die Literatur und Politik als eng verbunden betrachtet und die folgende Produktion H. Manns entscheidend bestimmt. Das zweideutige Licht, das H. Mann auf seinen «Tyrann » wirft wie auf die Fabel, die er entwickelt, beweist, dass er sich 1905 zwischen beiden Optiken bewegt, von denen die zweite die Oberhand gewinnen wird, ohne dass das grosse Vorbild jedoch je vollständig aufgegeben wurde.

\section{AUTEUR}

\section{CHANTAL SIMONIN}

Université Charles-de-Gaulle - Lille III 